\title{
III. SUB-BOTTOM DEPTHS OF INTERVALS CORED DURING LEGS 44 AND 44A
}

DSDP Staff

APPENDIX III

Sub-Bottom Depths of Intervals Cored During Legs 44 and 44A

\begin{tabular}{|c|c|c|c|c|c|c|}
\hline Hole & Core & $\begin{array}{l}\text { Top } \\
(\mathrm{m})\end{array}$ & $\begin{array}{l}\text { Bottom } \\
\text { (m) }\end{array}$ & $\begin{array}{l}\text { Recovery } \\
\text { (m) }\end{array}$ & $\begin{array}{c}\text { Last } \\
\text { Section } \\
\text { (No.) }\end{array}$ & $\begin{array}{l}\text { Length } \\
\text { of " } 0 \text { " } \\
\text { Section }\end{array}$ \\
\hline \multicolumn{7}{|c|}{ Leg 44} \\
\hline $\begin{array}{l}388 \\
388 \mathrm{~A}\end{array}$ & 1 & 0.0 & 8.5 & 4.2 & 4 & \\
\hline $388 \mathrm{~A}$ & 2 & 37.0 & 46.5 & 3.8 & 3 & \\
\hline $388 \mathrm{~A}$ & 3 & 46.5 & 53.5 & 0.15 & & \\
\hline $388 \mathrm{~A}$ & 4 & 208.0 & 217.5 & 0.8 & 1 & \\
\hline $388 \mathrm{~A}$ & 5 & 246.0 & 255.5 & 9.5 & 6 & 50 \\
\hline $388 \mathrm{~A}$ & 6 & 284.0 & 293.5 & 1.7 & 2 & \\
\hline $388 \mathrm{~A}$ & 7 & 293.5 & 300.5 & 5.0 & 4 & \\
\hline $388 \mathrm{~A}$ & 8 & 303.0 & 312.5 & 1.3 & 1 & \\
\hline $388 \mathrm{~A}$ & 9 & 312.5 & 322.0 & 7.0 & 6 & \\
\hline $388 \mathrm{~A}$ & 10 & 322.0 & 331.5 & 1.2 & 1 & \\
\hline $388 \mathrm{~A}$ & 11 & 331.5 & 341.0 & 7.8 & 6 & 50 \\
\hline 389 & 1 & 30.0 & 39.5 & 3.5 & 3 & \\
\hline 390 & 1 & 0.0 & 9.5 & 8.2 & 6 & \\
\hline 390 & 2 & 123.5 & 133.0 & 0.40 & 1 & \\
\hline 390 & 3 & 133.0 & 142.5 & 4.1 & 3 & \\
\hline 390 & 4 & 142.5 & 152.0 & 2.2 & 2 & \\
\hline 390 & 5 & 152.0 & 161.5 & 2.4 & 2 & \\
\hline 390 & 6 & 161.5 & 171.0 & 0.6 & 1 & \\
\hline 390 & 7 & 171.0 & 180.5 & 0.0 & & \\
\hline 390 & 8 & 180.5 & 190.0 & 7.7 & 6 & \\
\hline 390 & 9 & 190.0 & 199.5 & 1.6 & 1 & \\
\hline 390 & 10 & 199.5 & 206.0 & 0.05 & & \\
\hline $390 \mathrm{~A}$ & 1 & 9.5 & 19.0 & 1.2 & 1 & \\
\hline $390 \mathrm{~A}$ & 2 & 19.0 & 28.5 & 2.3 & 2 & \\
\hline $390 \mathrm{~A}$ & 3 & 28.5 & 38.0 & 4.8 & 4 & \\
\hline $390 \mathrm{~A}$ & 4 & 38.0 & 47.5 & 9.5 & 6 & 40 \\
\hline $390 \mathrm{~A}$ & 5 & 47.5 & 57.0 & 4.1 & 4 & \\
\hline $390 \mathrm{~A}$ & 6 & 57.0 & 66.5 & 9.5 & 6 & 40 \\
\hline $390 \mathrm{~A}$ & 7 & 66.5 & 76.0 & 5.3 & 4 & \\
\hline $390 \mathrm{~A}$ & 8 & 76.0 & 85.5 & 4.9 & 4 & \\
\hline $390 \mathrm{~A}$ & 9 & 85.5 & 95.0 & 1.3 & 1 & \\
\hline $390 \mathrm{~A}$ & 10 & 95.0 & 104.5 & 9.5 & 6 & 40 \\
\hline $390 \mathrm{~A}$ & 11 & 104.5 & 114.0 & 7.8 & 6 & \\
\hline $390 \mathrm{~A}$ & 12 & 114.0 & 123.5 & 9.5 & 6 & 45 \\
\hline $390 \mathrm{~A}$ & 13 & 123.5 & 133.0 & 9.5 & 6 & 40 \\
\hline $390 \mathrm{~A}$ & 14 & 133.0 & 142.5 & 7.6 & 5 & \\
\hline 391 & 1 & 0.0 & 4.5 & 2.3 & 2 & \\
\hline $391 \mathrm{~A}$ & 1 & 86.0 & 95.5 & 8.1 & 7 & \\
\hline $391 \mathrm{~A}$ & 2 & 31.5 & 41.0 & 5.0 & 4 & \\
\hline $391 \mathrm{~A}$ & 3 & 145.5 & 155.0 & 8.2 & 6 & \\
\hline $391 \mathrm{~A}$ & 4 & 202.5 & 212.0 & 6.2 & 4 & \\
\hline $391 \mathrm{~A}$ & 5 & 259.5 & 269.0 & 9.5 & 6 & 40 \\
\hline $391 \mathrm{~A}$ & 6 & 316.5 & 326.0 & 7.7 & 5 & \\
\hline $391 \mathrm{~A}$ & 7 & 326.0 & 335.5 & 7.2 & 5 & \\
\hline $391 \mathrm{~A}$ & 8 & 335.5 & 345.0 & 1.4 & 1 & \\
\hline $391 \mathrm{~A}$ & 9 & 354.5 & 364.0 & 9.5 & 6 & 50 \\
\hline $391 \mathrm{~A}$ & 10 & 373.5 & 383.0 & 9.5 & 6 & 40 \\
\hline $391 \mathrm{~A}$ & 11 & 411.5 & 421.0 & 8.7 & 6 & 50 \\
\hline $391 \mathrm{~A}$ & 12 & 468.5 & 478.0 & 9.6 & 6 & 50 \\
\hline $391 \mathrm{~A}$ & 13 & 525.5 & 535.0 & 9.5 & 6 & 50 \\
\hline $391 \mathrm{~A}$ & 14 & 535.0 & 544.5 & 0.01 & & \\
\hline $391 \mathrm{~A}$ & 15 & 544.5 & 554.0 & 0.05 & & \\
\hline $391 \mathrm{~A}$ & 16 & 554.0 & 563.5 & 2.2 & 2 & \\
\hline $391 \mathrm{~A}$ & 17 & 568.5 & 573.0 & 5.0 & 4 & \\
\hline $391 \mathrm{~A}$ & 18 & 573.0 & 582.5 & 0.1 & & \\
\hline $391 \mathrm{~A}$ & 19 & 582.5 & 592.0 & 5.2 & 4 & \\
\hline $391 \mathrm{~A}$ & 20 & 639.5 & 649.0 & 8.3 & 6 & \\
\hline
\end{tabular}

APPENDIX III - Continued

\begin{tabular}{|c|c|c|c|c|c|c|}
\hline Hole & Core & $\begin{array}{l}\text { Top } \\
\text { (m) }\end{array}$ & $\begin{array}{l}\text { Bottom } \\
\text { (m) }\end{array}$ & $\begin{array}{c}\text { Recovery } \\
\text { (m) }\end{array}$ & $\begin{array}{c}\text { Last } \\
\text { Section } \\
\text { (No.) }\end{array}$ & $\begin{array}{l}\text { Length } \\
\text { of " } 0 \text { " } \\
\text { Section }\end{array}$ \\
\hline
\end{tabular}

Leg 44 - Continued

$391 \mathrm{~A} \quad 21 \quad 649.0$

$\begin{array}{rrr}391 \mathrm{~B} & 1 & 0.0\end{array}$

$391 \mathrm{C} \quad 1 \quad 335.5$

$391 \mathrm{C} \quad 2 \quad 611.0$

$391 \mathrm{C} \quad 3 \quad 658.5$

$391 \mathrm{C} \quad 4 \quad 668.0$

$391 \mathrm{C} \quad 5 \quad 677.5$

$391 \mathrm{C} \quad 6 \quad 687.0$

391C $\quad 7 \quad 725.0$

$\begin{array}{lll}391 \mathrm{C} & 8 & 782.0 \\ 391 \mathrm{C} & 9 & 839.0\end{array}$

391C $\quad 9 \quad 839.0$

$\begin{array}{lll}391 \mathrm{C} & 10 & 896.0 \\ 391 \mathrm{C} & 11 & 924.5\end{array}$

391C $\quad 12 \quad 953.0$

391C $13 \quad 981.5$

391C $\quad 14 \quad 1000.5$

391C $15 \quad 1010.0$

391C $\quad 16 \quad 1019.5$

$\begin{array}{lll}391 \mathrm{C} & 17 & 1029.0\end{array}$

391C $\quad 18 \quad 1038.5$

391C $19 \quad 1048.0$

391C $\quad 20 \quad 1057.5$

391C $21 \quad 1086.0$

$\begin{array}{lll}391 C & 22 & 1095.5\end{array}$

391C $\quad 23 \quad 1114.5$

391C $24 \quad 1124.0$

$391 \mathrm{C} \quad 25 \quad 1133.5$

$\begin{array}{lll}391 \mathrm{C} & 26 & 1143.0 \\ 391 \mathrm{C} & 27 & 1152.5\end{array}$

391C $\quad 28 \quad 1162.0$

$391 \mathrm{C} \quad 29 \quad 1171.5$

$\begin{array}{lll}391 \mathrm{C} & 30 & 1181.0 \\ 391 \mathrm{C} & 31 & 1190.5\end{array}$

$\begin{array}{lll}391 \mathrm{C} & 31 & 1190.5 \\ 391 \mathrm{C} & 32 & 1200.0\end{array}$

$\begin{array}{lll}391 \mathrm{C} & 33 & 1209.5\end{array}$

$391 \mathrm{C} \quad 34 \quad 1219.0$

$\begin{array}{lll}391 \mathrm{C} & 35 & 1228.5\end{array}$

$391 \mathrm{C} \quad 36 \quad 1238.0$

$\begin{array}{lll}391 \mathrm{C} & 37 & 1247.5\end{array}$

391C $\quad 38 \quad 1257.0$

$\begin{array}{lll}391 C & 39 & 1266.5\end{array}$

$391 \mathrm{C} \quad 40 \quad 1276.0$

391C $41 \quad 1285.5$

391C $\quad 42 \quad 1295.0$

391C $\quad 43 \quad 1304.5$

$\begin{array}{lll}391 \mathrm{C} & 44 & 1314.0\end{array}$

$391 \mathrm{C}$

391C $46 \quad 1333.0$

$\begin{array}{lll}391 \mathrm{C} & 47 & 1342.5\end{array}$

$\begin{array}{lll}391 \mathrm{C} & 48 & 1352.0\end{array}$

391C $\quad 49 \quad 1361.5$

$391 \mathrm{C} \quad 50 \quad 1371.0$

$\begin{array}{lll}391 C & 51 & 1380.5\end{array}$

$391 C \quad 52 \quad 1390.0$

$391 \mathrm{C} \quad 53 \quad 1399.5$

$391 \mathrm{C} \quad 54 \quad 1409.0$

\begin{tabular}{lll}
392 & 1 & 47.5 \\
392 & 2 & 57.0 \\
\hline
\end{tabular}

$\begin{array}{rr}658.5 & 9.1 \\ 9.5 & 9.3\end{array}$

$\begin{array}{rr}9.5 & 9.3 \\ 345.0 & 3.1\end{array}$

$\begin{array}{rr}345.0 & 3.1 \\ 620.5 & 9.5\end{array}$

$\begin{array}{ll}668.0 & 0.3\end{array}$

$\begin{array}{ll}677.5 & 0.7\end{array}$

687.0

$696.5 \quad 9.5$

$734.5 \quad 2.0$

$791.5 \quad 2.2$

$848.5 \quad 4.2$

$905.5 \quad 3.9$

$934.0 \quad 4.7$

$962.5 \quad 8.6$

$\begin{array}{ll}991.0 & 0.2\end{array}$

$1010.0 \quad 3.8$

$1019.5 \quad 4.2$

$1029.0 \quad 5.6$

$1038.5 \quad 2.4$

$1048.0 \quad 1.1$

$1057.5 \quad 0.0$

$1067.0 \quad 0.40$

$1095.5 \quad 5.5$

$1104.0 \quad 0.05$

$1124.0 \quad 0.0$

$1133.5 \quad 8.5$

$1143.0 \quad 5.7$

$1152.5 \quad 5.0$

$1162.0 \quad 5.7$

$1171.5 \quad 4.7$

$1181.0 \quad 7.1$

$1190.5 \quad 5.1$

$1200.0 \quad 8.5$

$1209.5 \quad 5.1$

$1219.0 \quad 3.8$

$1228.5 \quad 5.1$

$1238.0 \quad 6.1$

1247.5

1257.0

1266.5

1276.0

1285.5

1295.0

1304.5

1314.0

1323.5

1333.0
1342.5

1352.0

1361.5

1371.0

1380.5

1385.5

1399.5

1409.0

412.0
57.0

\section{1}

2.0
6.0

6.3

6.3

6.1

7.1

5.9

8.0

4.2

1.3
1.0

1.9

1.70

1.3

1.7
5.0

0.03

$57.0-0.2$

$\begin{array}{ll}59.0 & 3.1\end{array}$
20

40

50

40

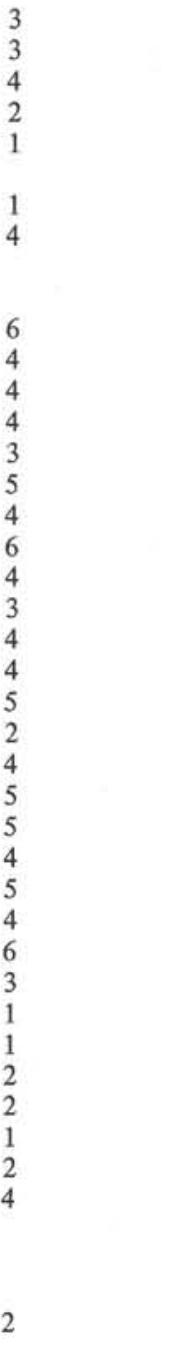


APPENDIX III - Continued

\begin{tabular}{|c|c|c|c|c|c|c|}
\hline Hole & Core & $\begin{array}{l}\text { Top } \\
\text { (m) }\end{array}$ & $\begin{array}{l}\text { Bottom } \\
(\mathrm{m})\end{array}$ & $\begin{array}{l}\text { Recovery } \\
\text { (m) }\end{array}$ & $\begin{array}{c}\text { Last } \\
\text { Section } \\
\text { (No.) }\end{array}$ & $\begin{array}{l}\text { Length } \\
\text { of "0" } \\
\text { Section }\end{array}$ \\
\hline \multicolumn{7}{|c|}{ Leg 44 - Continued } \\
\hline $392 \mathrm{~A}$ & 1 & 50.5 & 60.0 & 2.7 & 2 & \\
\hline $392 \mathrm{~A}$ & 2 & 66.0 & 69.5 & 3.5 & 3 & \\
\hline $392 \mathrm{~A}$ & 3 & 79.0 & 88.5 & 3.7 & 3 & \\
\hline $392 \mathrm{~A}$ & 4 & 88.5 & 98.0 & 0.6 & 1 & \\
\hline $392 \mathrm{~A}$ & 5 & 98.0 & 107.5 & 1.7 & 2 & \\
\hline $392 \mathrm{~A}$ & 6 & 107.5 & 117.0 & 0.9 & 1 & \\
\hline $392 \mathrm{~A}$ & 7 & 117.0 & 126.5 & 0.2 & 1 & \\
\hline $392 \mathrm{~A}$ & 8 & 126.5 & 136.0 & 0.2 & 1 & \\
\hline $392 \mathrm{~A}$ & 9 & 136.0 & 139.0 & 1.5 & 1 & \\
\hline $392 \mathrm{~A}$ & 10 & 139.0 & 145.5 & 1.6 & 2 & \\
\hline $392 \mathrm{~A}$ & 11 & 145.5 & 155.0 & 0.95 & 1 & \\
\hline $392 \mathrm{~A}$ & 12 & 155.0 & 164.5 & 0.1 & & \\
\hline $392 \mathrm{~A}$ & 13 & 164.5 & 174.0 & 0.1 & & \\
\hline $392 \mathrm{~A}$ & 14 & 174.0 & 176.0 & 0.1 & & \\
\hline $392 \mathrm{~A}$ & 15 & 176.0 & 183.5 & 0.3 & 1 & \\
\hline $392 \mathrm{~A}$ & 16 & 183.5 & 193.0 & 0.9 & 1 & \\
\hline $392 \mathrm{~A}$ & 17 & 193.0 & 202.5 & 1.0 & 1 & \\
\hline $392 \mathrm{~A}$ & 18 & 202.5 & 212.0 & 0.1 & & \\
\hline $392 \mathrm{~A}$ & 19 & 212.0 & 221.5 & 0.8 & 1 & \\
\hline $392 \mathrm{~A}$ & 20 & 221.5 & 231.0 & 0.9 & 1 & \\
\hline $392 \mathrm{~A}$ & 21 & 231.0 & 240.5 & 0.7 & 1 & \\
\hline $392 \mathrm{~A}$ & 22 & 240.5 & 250.0 & 0.7 & 1 & \\
\hline
\end{tabular}

APPENDIX III - Continued

\begin{tabular}{|c|c|c|c|c|c|c|}
\hline Hole & Core & $\begin{array}{l}\text { Top } \\
\text { (m) }\end{array}$ & $\begin{array}{l}\text { Bottom } \\
\text { (m) }\end{array}$ & $\begin{array}{l}\text { Recovery } \\
\text { (m) }\end{array}$ & $\begin{array}{l}\text { Last } \\
\text { Section } \\
\text { (No.) }\end{array}$ & $\begin{array}{l}\text { Length } \\
\text { of "0" } \\
\text { Section }\end{array}$ \\
\hline
\end{tabular}

392A 24

392A 25

392A 26

392A 27

392A 28

392A 29

$392 \mathrm{~A} \quad 30$

392A 31

392A 32

392A $\quad 33$

$\begin{array}{ll}393 & 1 \\ 393 \mathrm{~A} & 1\end{array}$

Leg 44A

394

394 A

$394 \mathrm{~A}$

$394 \mathrm{~A}$

$394 \mathrm{~A}$

$394 \mathrm{~A}$

$394 \mathrm{~A}$

250.0
259.5
269.0
278.5
288.0
297.5
307.0
316.5
326.0
335.5
345.0
0.0
0.0

259.3

269.0

278.5

288.0

297.5

307.0

316.5

326.0

335.5

345.0

349.0

8.0

58.5

$\begin{array}{ll}0.7 & 1 \\ 0.2 & \\ 0.2 & \\ 0.6 & \\ 0.1 & \\ 0.4 & \\ 0.1 & \\ 0.1 & \\ 0.2 & \\ 0.0 & \\ 0.0 & \\ 4.4 & \\ 9.63 & 6\end{array}$

1

6

4

98.5
108.0
184.0
193.5
288.5

364.5

$\begin{array}{ll}3.2 & 2 \\ 0.5 & 1 \\ 0.3 & 1 \\ 0.1 & \\ 4.10 & 3 \\ 9.6 & 6\end{array}$

45 